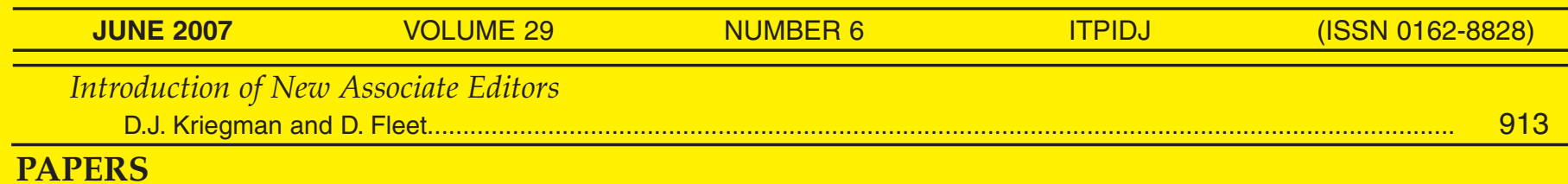

\title{
PAPERS
}

\section{Face and Gesture}

Dynamic Texture Recognition Using Local Binary Patterns with an Application to Facial Expressions

G. Zhao and M. Pietikäinen..

Image Segmentation and Feature Extraction

Toward Objective Evaluation of Image Segmentation Algorithms

R. Unnikrishnan, C. Pantofaru, and M. Hebert.

Curve/Surface Representation and Evolution Using Vector Level Sets with Application to the Shape-Based

Segmentation Problem

H.E. Abd El Munim and A.A. Farag

\section{Matching Registration}

Matching by Linear Programming and Successive Convexification

H. Jiang, M.S. Drew, and Z.-N. Li

\section{Motion Tracking and Analysis}

Model-Based Tracking by Classification in a Tiny Discrete Pose Space

L. Shang, P. Jasiobedzki, and M. Greenspan.....

\section{Morphological Filtering}

Mask-Based Second-Generation Connectivity and Attribute Filters

G.K. Ouzounis and M.H.F. Wilkinson.

\section{Object Recognition}

Discriminative Learning and Recognition of Image Set Classes Using Canonical Correlations

T.-K. Kim, J. Kittler, and R. Cipolla..

Recognition of Pornographic Web Pages by Classifying Texts and Images

W. Hu, O. Wu, Z. Chen, Z. Fu, and S. Maybank.

\section{Pattern Analysis and Classification}

Iterative RELIEF for Feature Weighting: Algorithms, Theories, and Applications

$$
\text { Y. Sun.... }
$$

\section{Stereo}

MonoSLAM: Real-Time Single Camera SLAM

A.J. Davison, I.D. Reid, N.D. Molton, and O. Stasse

Stereo Correspondence with Occlusion Handling in a Symmetric Patch-Based Graph-Cuts Model

(Contents continued on back cover) 